\title{
Heroin-Induced Ulcer Mimicking Pyoderma Gangrenosum
}

Deep Patel, BS ${ }^{1}$, Jennifer Mardini, BS ${ }^{1}$, Christopher Chu, MD², Max Disse, MD² $^{2}$ Alexandra

Flamm, MD ${ }^{2}$

${ }^{1}$ Penn State College of Medicine, Hershey, PA

${ }^{2}$ Department of Dermatology, Penn State Hershey Medical Center, Hershey, PA

\section{ABSTRACT}

Subcutaneous injections of illicit substances may be performed by some patients citing several advantages by the user, and can present to clinic with ulcers that clinically mimic pyoderma gangrenosum. However, it is vital to recognize heroin-induced ulcers and treat urgently to preserve function. Treatment requires a multidisciplinary approach with general medicine, dermatology, plastic surgery, wound care, infectious disease and addiction medicine. We present a patient with lower extremity ulceration due to subcutaneous injection of heroin which resembled PG.

\section{INTRODUCTION}

"Skin popping" is a term used to describe repetitive subcutaneous injections with illicit substances such as heroin and cocaine ${ }^{1}$. "Skin popping" is often due to extravasation of chemicals in the drug and can cause the formation of secondary tissue lesions, including thrombophlebitis, ulcerations, gangrene, and necrosis at and around the injection sites and can quickly evolve to limb amputation and death ${ }^{2,3}$.

Initial dermatological manifestations of "skin popping" may present similar to more common etiologies such as pyoderma gangrenosum (PG); however, all injectioninduced dermatological manifestations should be recognized and treated urgently to preserve function. Information and reports in the dermatologic literature are sparse, but is becoming increasing relevant. We present a patient with lower extremity ulceration due to subcutaneous injection of heroin which resembled PG.

\section{CASE REPORT}

A 29-year-old female with a history of intravenous and subcutaneous drug abuse presented for evaluation of a large skin ulcer on the left lower extremity. The skin manifestations began nine months prior when the patient injected heroin into her bilateral lower extremities. She subsequently developed an enlarging inflammatory nodule and ulcers at injection points of both her right thigh and left lower extremity, which were treated with irrigation and debridement. The patient reports that her right thigh wound healed well; however, the wound on her left lower extremity persisted and seems to have worsened after her debridement and multiple subsequent antibiotic courses.

Several cultures were previously obtained, which revealed 1+ Pseudomonas aeruginosa, sensitive to ciprofloxacin. Although the cultures showed $\mathrm{P}$. aeruginosa colonization, pointing to ecthyma gangrenosum, the etiology of the ulcer was

July 2021 Volume 5 Issue 4 
considered not due to chronic infection as four courses of ciprofloxacin treatment did not improve the skin lesion. The nonhealing wound has been treated with a variety of wound care modalities such as absorbent dressings with compression wraps with no improvement. The multi-disciplinary efforts for this patient included dermatology, infectious disease for microbial etiologies, rheumatology for systemic disease workup and vascular surgery for wound care.

On physical exam, a $10 \times 15 \mathrm{~cm}$ welldemarcated deep ulcer with a beefy red, fibrinous base containing visible subcutaneous and granulation tissue and a rim of erythema and areas of undermined edges on the left lower extremity was observed (Figure 1). The arms and legs had numerous well-healed hyperpigmented macules and patches, where patient notes past injection sites. Of note, patient was afebrile and had a normal white blood cell count.

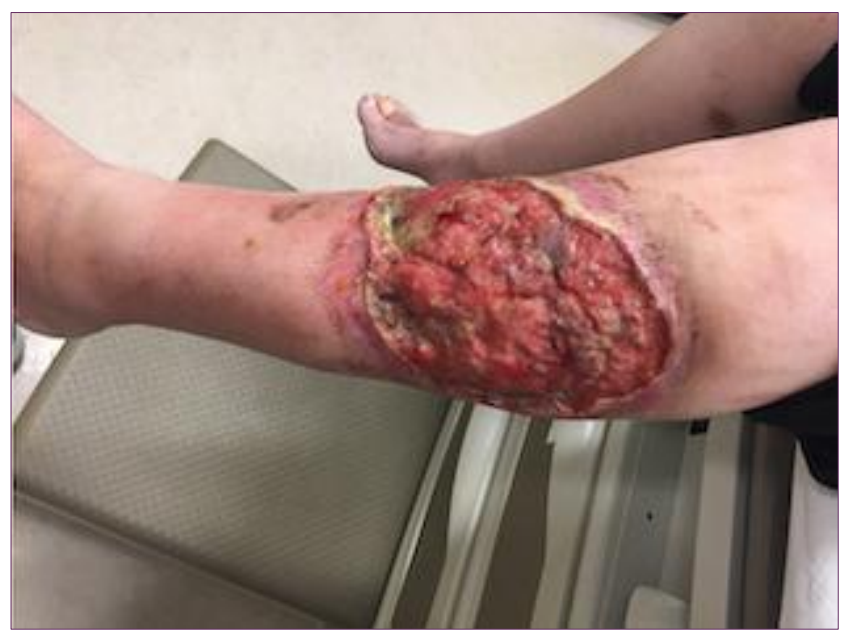

Figure 1.

Differential diagnosis included vasculitis, Hepatitis B, superficial ulcerating rheumatoid necrobiosis however lab evaluation for systemic disease was negative. Given the history of the worsening skin lesion after debridement and the characteristic appearance, the wound was biopsied by dermatology to assess for pyoderma gangrenosum (PG). Punch biopsy at the edge of the ulcer revealed perivascular lymphoplasmacytic infiltrate without characteristic dense neutrophilic infiltrate of PG, and histopathology did not coordinate with PG (Figure 2). Tissue culture was also obtained for anaerobe, fungus, bacteria, and acid-fast bacilli, which were negative except one isolated colony of $P$. aeruginosa.

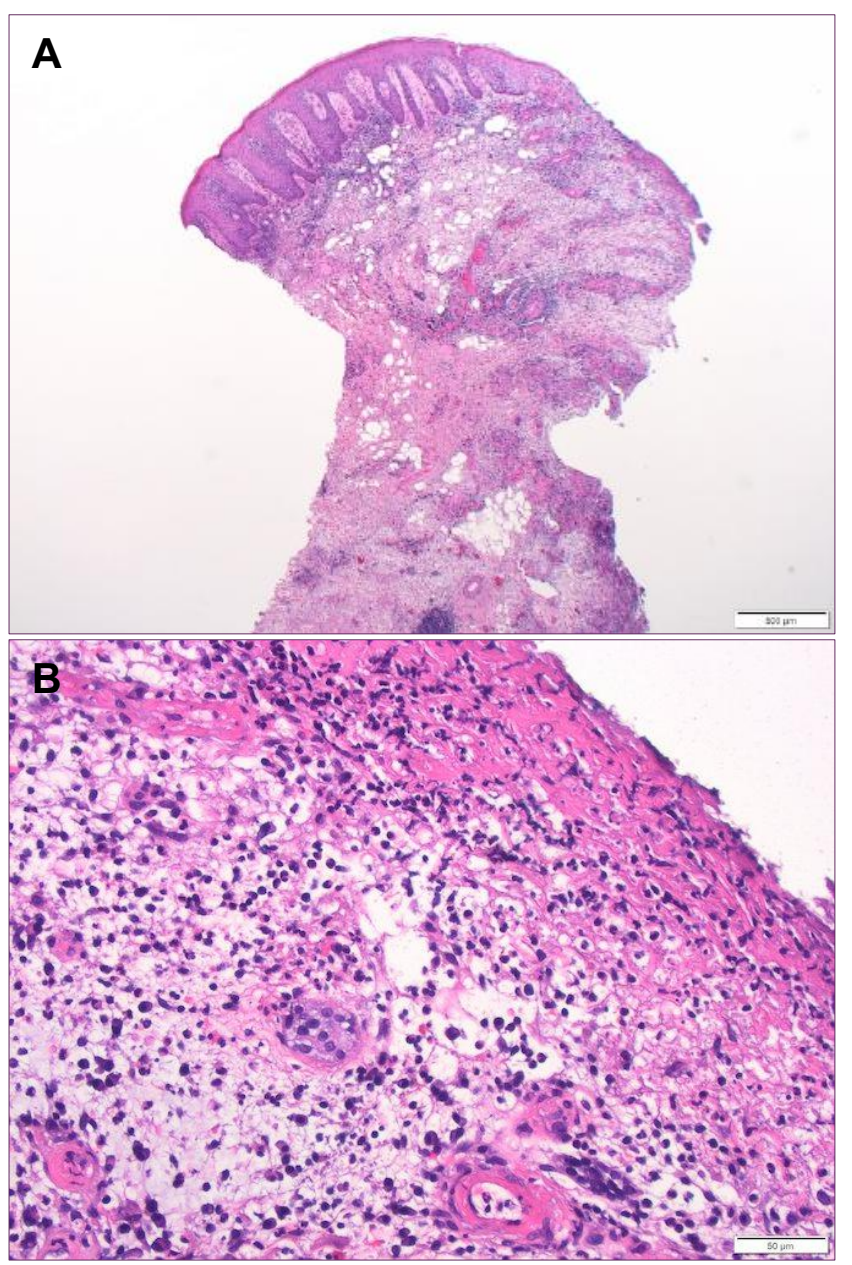

Figure 2. A, B

Patient was offered addiction medicine consultation, which she declined. She agreed to follow up and treatment with wound center with local wound care and debridement. Follow up at the wound clinic one month after treatment revealed an improvement in ulcer size and appearance (Figure 3). Patient is

July 2021 Volume 5 Issue 4 
currently on Suboxone and reported that she is no longer injecting heroin. She had no signs of subcutaneous injections.

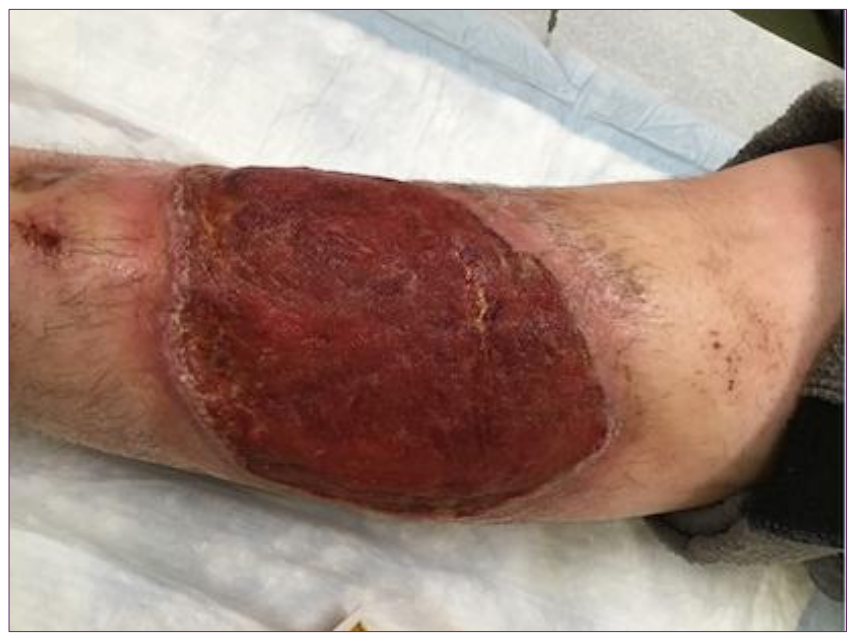

Figure 3.

\section{DISCUSSION}

Our patient was diagnosed with a heroin induced ulcer secondary to subcutaneous injection. Because of extravasation of chemicals in the drugs, subcutaneous injection of heroin and other illicit substances can result in ulcerations, gangrene, and necrosis to local tissue causing it to clinically resemble $\mathrm{PG}^{2,3}$. For example, krokodil, a synthetic addictive substitute for heroin, appears to be associated with necrotic and gangrenous tissue destruction more than other substances [4]. The presence of gasoline and $\mathrm{HCl}$ in krokodil induces discolored scale-like skin and ulceration, while venous extravasation of heroin leads to extensive necrosis of the skin and subcutaneous layer ${ }^{4}$. The evolving nature of these exogenous-induced ulcers, however, may lead to limb amputation and death ${ }^{5}$. Therefore, the importance of a broad differential diagnosis and confirmatory biopsy of the lesion is critical for cases resembling PG, especially in high risk patient populations.
Notably, other processes have been reported to mimic PG as well including bromoderma, cutaneous diphtheria, cutaneous cryptococcus, granulomatosis with polyangiitis, and metastatic Crohn disease ${ }^{5-9}$. This further highlights the importance for a broad initial differential diagnosis in cases clinically resembling PG.

In the present case, dermatology was consulted to assess for $P G$ as the clinical findings were consistent with this condition. The dermatopathology of heroin ulcers have been found to show nonspecific findings such as leukocytoclastic vasculitis, dermal pigment deposition, non-specific ulceration/scarring and necrobiosis lipoidicalike dermatitis ${ }^{10}$. Our case revealed perivascular lymphoplasmacytic infiltrate. More importantly, the characteristic dense and required neutrophilic infiltrates of $P G$ were absent. Along with the patient's history of subcutaneous injection of heroin, a diagnosis of heroin-induced ulcer was made.

\section{CONCLUSION}

Increasing use of heroin and other illicit substances in our patients make it important for dermatologists to be aware of the cutaneous manifestations caused by subcutaneous injections of illicit drugs. Our patient volunteered her drug use, however, many will not and thus it is important to be suspicious in high risk patients presenting with early manifestations of injection induced ulcerations, which may resemble other diseases such as pyoderma gangrenosum.

Treatment of these wounds will require a multidisciplinary approach including dermatology, infectious disease, wound care, plastic surgery and internal medicine. Because of poor compliance, being lost to 
follow up and the natural history of this disease, these patients are at a higher risk for complications.

Conflict of Interest Disclosures: None

Funding: None

Corresponding Author:

Deep Patel, BS

500 University Ave

Hershey PA 17033

Phone: 717-531-5898

Fax: 717-531-4702

Email: dpatel3@pennstatehealth.psu.edu
10. Tse JY, Adisa M, Goldberg LJ, et al. Dermatopathologic manifestations of intravenous drug use. J Cutan Pathol. 2015;42(11):815-823.

\section{References:}

1. Alvi A, Ravichandran D. An unusual case of breast ulceration. Breast. 2006;15(1):115-116.

2. Onesti MG, Fioramonti $P$, Fino $P$, et al. Skin ulcer caused by venous extravasation of heroin. Int Wound J. 2014;11(4):409-411.

3. Alves EA, Grund JP, Afonso CM, et al. The harmful chemistry behind krokodil (desomorphine) synthesis and mechanisms of toxicity. Forensic Sci Int. 2015;249:207-213.

4. Haskin, A., Kim, N., \& Aguh, C. (2016). A new drug with a nasty bite: A case of krokodilinduced skin necrosis in an intravenous drug user. JAAD case reports, 2(2), 174-176. https://doi.org/10.1016/j.jdcr.2016.02.007

5. Oda F, Tohyama M, Murakami A, et al. Bromoderma mimicking pyoderma gangrenosum caused by commercial sedatives. J Dermatol. 2016;43(5):564-566.

6. Morgado-Carrasco D, Riquelme-Mc Loughlin C, Fustá-Novell X, et al. Cutaneous Diphtheria Mimicking Pyoderma Gangrenosum. JAMA Dermatol. 2018;154(2):227-228.

7. Kindle SA, Camilleri MJ, Gibson LE, et al. Granulomatosis with polyangiitis mimicking classic inflammatory bowel disease-associated pyoderma gangrenosum. Int J Dermatol. 2017;56(1):e1-e3.

8. Kikuchi N, Hiraiwa T, Ishikawa M, et al. Cutaneous Cryptococcosis Mimicking Pyoderma Gangrenosum: A Report of Four Cases. Acta Derm Venereol. 2016;96(1):116-117.

9. Avilés-Izquierdo JA, Suárez-Fernández R, Lázaro-Ochaita $\mathrm{P}$, et al. Metastatic Crohn's disease mimicking genital pyoderma gangrenosum in an HIV patient. Acta Derm Venereol. 2005;85(1):60-62. 